\title{
Warum wenden sich Ärzte der Homöopathie zu?
}

\author{
Von Daniel Walther
}

\section{Zusammenfassung}

Der Beitrag liefert einen Überblick über die Gründe und persönlichen Motive von Ärzten und Heilpraktikern, die sich für die Homöopathie entschieden haben. Grundlage sind 26 biografische Kurzberichte. Nach einer kurzen Darstellung der Sozialstruktur werden die geschilderten Beweggründe 4 Kategorien zugeteilt. Am häufigsten wandten sich die Autoren wegen ihrer Unzufriedenheit mit der Schulmedizin der Homöopathie zu. Fast ebenso viele wurden selbst oder ihre Kinder mit homöopathischen Arzneimitteln erfolgreich behandelt. Anziehend wirkten darüber hinaus Heilerfolge durch die Homöopathie, deren Zeuge die Autoren geworden sind. Einige gaben letztlich ihr Selbstverständnis als ganzheitlich denkender und behandelnder Arzt als Hauptgrund an.

\section{Summary}

This article provides a overview of the individual motives of physicians and non-medical practitioners, which integrated homeopathy in their medical practice. The basis for this examination are 26 biographical summaries. After a short presentation of the social structure the narrated motives will be attached to 4 categories. Most frequently the writers turned to homeopathy because of their dissatisfaction with orthodox medical treatment. Almost the same amount of physicians/non-medical practitioners or their children have been ill and were cured with homeopathic remedies. Some homeopaths also were attracted by witnessed successful homeopathic treatments. Above all there are several writers, which boasted their selfconcept as a holistic doctor as main reason why they switched to homeopathy.

\section{Schlüsselwörter}

Homöopathie, Schulmedizin, Kritik, Ärzte, Heilpraktiker, Motive, Entscheidung

\section{Keywords}

Homeopathy, academic medicine, criticism, physicians, non-medical practitioner, motives, decision

\section{Einleitung}

„Hier bin ich in der Suche nach ,meiner" Medizin angekommen“ - Das könnte die Aussage eines Patienten ${ }^{1}$ sein, der nach frustrierenden Erfahrungen mit erfolglosen konventionellen Behandlungsmethoden zur Homöopathie gefunden hat. Zahlreiche Studien belegen die anhaltende Popularität und Nachfrage alternativmedizinischer Angebote (vgl. [2,7]) und machen deutlich, dass immer mehr Menschen Mitsprache verlangen, wenn es um die Wiederherstellung ihrer Gesundheit geht. Entgegen dieser spontanen Vermutung stammt die eingangs zitierte Aussage jedoch nicht von einem mündigen und reflektierten Patienten, dem Exponenten der gegenwärtigen „Gesundheitsgesellschaft“ (vgl. [3]), sondern von einer ärztlichen Homöopathin. Ihre Schilderungen sind Teil eines Samples von 26 autobiographischen Texten, in denen nicht Patienten, sondern Ärzte und Heilpraktiker über die näheren Umstände ihrer Hinwendung zur Homöopathie berichten. Besonders aussagekräftige Passagen dieser biographischen Kurzberichte werden aus Datenschutzgründen anonymisiert wiedergegeben. Um sie dennoch zuordnen zu können, werden sie mit dem Kürzel „SH“ (Sample Homöopathen) und der entsprechenden Bearbeitungsnummer versehen.

Die Texte entstanden im Zeitraum zwischen 2010 und 2013, nachdem das Institut für Geschichte der Medizin der Robert Bosch Stiftung Stuttgart (IGM) einen entsprechenden Aufruf zur Niederschrift jener Beweggründe in der Allgemeinen Homöopathischen Zeitung veröffentlichte. ${ }^{2}$ Ziel des Aufrufs sollte - analog zu patientensoziologischen Fragestellungen - die Erforschung der Motive sein, die einerseits das medizinische Fachpersonal dazu bewogen haben, mit den durch das Medizinstudium angeeigneten, herrschenden naturwissenschaftlichen Denkmodellen zu brechen. Andererseits spürt der Artikel den Motiven von medizinischen Laien nach, die beruflich zunächst einen völlig anderen Weg eingeschla- 
gen, sich trotz potenzieller Existenzrisiken dann aber doch für die Ausbildung zum homöopathisch orientierten Heilpraktiker entschieden haben.

\section{Die Autoren der Kurzbiographien}

Zentraler Bestandteil der Erhebung ist neben den Kurzberichten ein standardisierter Fragebogen, der die wesentlichen biographischen Eckdaten der Autoren erfasst. Von den 26 Ärzten und Heilpraktikern, die dem Aufruf des IGM nachgekommen sind und ihren persönlichen Weg zur Homöopathie zum Teil sehr ausführlich in Worte fassten, sind 12 männlich (46\%) und 14 weiblich (54\%). 17 (65\%), also mehr als die Hälfte, haben Humanmedizin studiert, 3 weibliche und 4 männliche Autoren (27\%) praktizieren die Homöopathie als niedergelassene Heilpraktiker ohne medizinisch-akademische Vorbildung und 2 Homöopathen (8\%), eine Frau und ein Mann, arbeiten hauptberuflich als Zahnmediziner.

Betrachtet man das Geschlechterverhältnis nur unter den Medizinern, so fällt der Gender-Gap deutlicher aus. Unter den 19 teilnehmenden Human- und Zahnmedizinern befinden sich 11 Frauen (58\%) und 8 Männer (42\%). Die Aufteilung der Geschlechter kommt damit den aktuellen absoluten Zahlen innerhalb der homöopathischen Ärzteschaft nahe. Eine Nachfrage beim Deutschen Zentralverein homöopathischer Ärzte (DZVhÄ) ergab, dass - nach Datenbestand des DZVhÄ vom Januar 2017 $63 \%$ der Ärzte mit Zusatzbezeichnung Homöopathie und/ oder dem Homöopathie-Diplom des DZVhÄ weiblich sind. Nur etwas mehr als ein Drittel (37\%) der anerkannten ärztlichen Homöopathen in Deutschland ist männlich.

Sowohl die Umfragen über die Inanspruchnahme homöopathischer Arzneimittel (vgl. [8, 9]) als auch die Mitgliederstatistik ${ }^{3}$ des Deutschen Verbands für Homöopathie und Lebenspflege Hahnemannia zeigen, dass mehr Frauen als Männer an Homöopathie interessiert sind. In Anbetracht der spezifischen Geschlechterverteilung innerhalb der homöopathischen Ärzteschaft trifft dies also auch auf die Mehrheit der professionellen Homöopathen zu. Vergleicht man die Zahlen mit der offiziellen Statistik der Bundesärztekammer, so kehrt sich das Geschlechterverhältnis allerdings ins Gegenteil: Ende 2016 waren in Deutschland 175901 Ärztinnen und 202706 Ärzte berufstätig. Der Männeranteil überwiegt demnach leicht mit 54\% [10]. In Fachgebieten wie Kinder- und Jugendmedizin, Innerer Medizin und Allgemeinmedizin, die auch Homöopathen bevorzugt ausüben, dominieren ebenfalls Männer. Besonders groß ist der Unterschied zwischen männlichen und weiblichen Internisten. Von den insgesamt 52158 Ärzten für Innere Medizin ist weit mehr als die Hälfte männlich (m: 33 121=64\%; w: 19037 $=36 \%$ ). Als Allgemeinmediziner praktizieren insgesamt
43618 Ärzte, 20485 Frauen (47\%) und 23133 Männer (53\%). Lediglich in der Pädiatrie waren laut Bundesärztestatistik Ende 2015 erwartungsgemäß mehr Ärztinnen tätig, nämlich 8412 (58\%) gegenüber 6054 Kinderärzten (42\%).

Wie die ärztlichen Homöopathen haben auch 6 der 7 Heilpraktiker des Samples einen akademischen Hintergrund. Zwei von ihnen studierten Theologie bzw. Religionspädagogik, zwei weitere Biologie und Physik und eine Heilpraktikerin ist examinierte Erziehungswissenschaftlerin. Ein Heilpraktiker behandelte als promovierter Veterinärmediziner lange Jahre Tiere mit homöopathischen Mitteln. Nachdem ihn immer häufiger deren Besitzer nach Ratschlägen bezüglich einer homöopathischen Behandlung ihrer eigenen Beschwerden befragten, entschied er sich zur Homöopathie- und HeilpraktikerAusbildung.

Schaut man sich die Spezialisierung der Ärzte näher an, so fällt auf, dass mit Ausnahme der Zahn- und Tierärzte die Mehrzahl der approbierten Homöopathen niedergelassene Haus- und Kinderärzte sind. Das dürfte zum einem mit dem breiten Krankheitspanorama zusammenhängen, mit dem diese Ärzte in ihrer Praxis täglich konfrontiert werden. Die Homöopathie stellt hier eine Alternative oder Ergänzung dar, vor allem in leichteren Fällen oder bei chronischen bzw. rezidiven Erkrankungen. Zum anderen stehen viele Haus- und Kinderärzte einer „ganzheitlichen“ Betrachtung der Beschwerden ihrer Patienten offen gegenüber. Hier bietet sich die Homöopathie ebenfalls an, da eine umfassende Anamnese, bei der die individuellen Lebensumstände und -gewohnheiten eine zentrale Rolle spielen, elementarer Bestandteil der Therapie ist.

\section{Die Einteilung der Beweggründe in Kategorien}

Bei der Auswertung der autobiographischen Kurzberichte zeichnete sich schnell ab, dass bestimmte Beweggründe häufiger genannt werden. Zur besseren Übersicht ordne ich diese Gründe insgesamt 4 Kategorien zu, wobei die Grenzen fließend und die jeweiligen Motive nicht immer trennscharf zu unterscheiden sind. In die Kategorie „Unzufriedenheit mit der konventionellen Medizin“" (Kategorie I) gehören beispielsweise das Angewiesensein auf stark wirkende Synthetika oder die Objektivierung des Patienten. Konnte sich ein Homöopath von der Heilkraft der Homöopathie am eigenen oder am Leib seiner Kinder überzeugen, so fallen die Gründe seiner Konversion in die Kategorie „Unmittelbare Krankheitserfahrung“ (Kategorie II). Die beiden anderen Kategorien lauten „Schlüsselerlebnis, Initialmoment“ (Kategorie III) sowie „Selbstverständnis als Arzt und Heilpraktiker“ (Kategorie IV). Letzterer ist das eingangs zitierte Beispiel jener ärztlichen 
Homöopathin zuzuteilen, die das Ende der Suche nach der, ihrer Meinung nach, richtigen Heilmethode mit den Worten: „So möchte ich Arzt sein, so möchte ich Patienten begegnen, mit ihnen arbeiten und ihnen helfen" (SH 13) untermauert.

\section{Kategorie I: Unzufriedenheit mit der konventionellen Medizin}

Diejenigen Homöopathen, die sich aus Unzufriedenheit mit der konventionellen Medizin und/oder Kritik an deren monokausalen Therapiekonzepten für die ganzheitlich ausgerichtete Homöopathie entschieden haben, bilden im Sample die größte Gruppe. Insgesamt 11 Autoren (42\%) geben entsprechende Gründe an. Dass es sich dabei fast ausschließlich um Ärzte handelt, dürfte wohl damit zusammenhängen, dass sie die Missstände des Gesundheitswesens am unmittelbarsten wahrnehmen; mitunter sogar doppelt als behandelnde Ärzte und Hilfe suchende Patienten bzw. Eltern. Entsprechende Gründe werden allerdings auch von Heilpraktikern vorgebracht. Die durchschnittlich 8 bzw. bei Ärzten 7 Jahre dauernde Zeitspanne zwischen Studienabschluss und dem Zeitpunkt der definitiven Hinwendung zur Homöopathie ist ein Indiz für das wachsende Unbehagen, das entweder schon während des Medizinstudiums oder unmittelbar nach Beginn der Tätigkeit als approbierter Arzt empfunden wird. So fing beispielsweise eine niedergelassene Kinderärztin nach 15 Jahren Praxistätigkeit an, sich nach anderen Möglichkeiten umzusehen, da sie wegen der ständig sich wiederholenden Krankheiten bei Kindern sehr frustriert gewesen sei (SH 22). Die Homöopathie stellt mit ihrem ganzheitlichen psychophysischen Verständnis von Gesundheit und Krankheit einen Ausweg aus dieser prekären Lage und eine Alternative zum in sich geschlossenen, naturwissenschaftlichen und ökonomisierten Medizinsystem dar.

\section{"Gescheit und gut"}

Besonders aufschlussreich und exemplarisch ist die Biographie einer 1950 geborenen österreichischen Ärztin. Mit 18 Jahren nahm sie das Medizinstudium in Wien auf, nachdem sie schon seit früher Kindheit den innigen Wunsch hegte, in Not geratenen Menschen zu helfen. Das Studium an sich sei ihr leicht gefallen, mit der Medizin im eigentlichen Sinne habe sie aufgrund der mangelnden Berücksichtigung der individuellen Beschwerden des Kranken hingegen nichts anfangen können. Das Schlüsselerlebnis, das sie endgültig mit der Schulmedizin brechen ließ, war die im Rahmen einer Vorlesung von einem Internisten vorgetragene Fallgeschichte:
„Er berichtete ausführlich über eine Patientin, die gerade auf seiner Klinik mit der Diagnose Hyperthyreose mit den gefährlichen Thyreostatica behandelt wurde [...]erfolgreich seinen Schilderungen gemäß. Er führte akribisch aus, wie diese Patientin wegen ihrer Nervosität und der Schlafstörungen seit 20 Jahren viele Ärzte aufgesucht hatte: Immer bekam sie Tranquilizer. Sie schlitterte immer weiter in ihre Krankheit hinein. Und jetzt wurde die klinische Diagnose gefunden: Hyperthyreose. Prof. D. feierte an dieser Geschichte den Erfolg der modernen Medizin.

Für mich war es ein Armutszeugnis: Die Patientin hatte ihre Ärzte jahrzehntelang um Hilfe gebeten und ihnen die Chance gegeben, ihr zunehmendes Leiden zum Guten zu wenden. Niemand hatte ihr ernsthaft helfen können, bis sie sich nun mit einer ,echten' Krankheit und Diagnose und schlussendlich einer aggressiven Therapie mit voraussichtlicher Defektheilung oder Dauermedikation abfinden muss.

Was ist das für eine Logik? Was ist das für ein Erfolg? Nein, eine solche Medizin will ich nicht.“(SH 13)

Diese Erkenntnis markierte folgerichtig den „Nullpunkt“ ihrer Studentenkarriere. Anfangs dachte sie an einen Abbruch des Studiums, fand dann im Klima der 68erBewegung aber Anschluss an Kommilitonen, die ihre Medizinkritik teilten. Bei einer Studenteninitiative kam sie erstmals in Berührung mit der Homöopathie. Was sie darüber hörte, „klang gescheit und gut“ (SH 13) und bewog sie, in einem Krankenhaus für Naturheilweisen in München zu famulieren. Dort habe sie erste Eindrücke „einer anderen, sorgfältigen patientenbezogenen Medizin mit dem reichen Schatz an phytotherapeutischen, diätetischen und physikalischen Maßnahmen “ (SH 13) sammeln können. In Wien überzeugte sie dann ein erfahrener Arzt vollends von der homöopathischen Heilmethode, indem er die Bedeutung nonverbaler Ausdrucksweisen der Menschen betonte und die Aufmerksamkeit auf deren Gestik, Benehmen und Verhalten lenkte. Das habe ihren Angaben zufolge wesentlich dazu beigetragen, ihren „einseitig geschulten Verstand wieder den Lebensphänomenen und dem realen Sein der Patienten gegenüber zu öffnen“ (SH 13). Die Homöopathie entspreche mit dieser Akzentuierung am ehesten ihrer Vision einer patientennahen und biographisch orientierten Medizin.

\section{„Aus purer Verzweiflung“}

Nicht aus „ideologischen Gründen“, sondern „aus purer Verzweiflung über die limitierten Heilungsmöglichkeiten der Schulmedizin" hat sich ein in einer ländlichen Region praktizierender Hausarzt für die Homöopathie entschieden. Er gibt in seiner Zuschrift an, er habe alle Patienten, wie auf der Universität und in der Klinik gelernt, zunächst schulmedizinisch behandelt. Nach 6-jähriger Pra- 
xistätigkeit sei er jedoch an den kleinen Kindern verzweifelt, die an rezidiven Mandel- und Ohrentzündungen litten und von ihm mit Antibiotika behandelt wurden. Das brachte langfristig aber keine Heilung. Die jungen Patienten seien stattdessen immer „hinfälliger“ (SH 26) geworden. Bei Neurodermitis verordnete er ausschließlich cortisonhaltige Salben, womit er ebenfalls nicht zufrieden gewesen sei, da das Hormon die Symptome nur verschlimmerte.

Schließlich erinnerte er sich an seine Mutter, die ihm im Kindesalter oft Schüßler-Salze gegeben hatte, wenn er krank gewesen war. Nach ersten Versuchen bei grippalen Infekten schöpfte er so viel Vertrauen, dass er einen Homöopathie-Kurs absolvierte. Dieser Kurs sei für ihn aber äußerst verwirrend gewesen, da er ,diese komische Sprache der Homöopathen nicht" (SH 26) verstand und sie sogar Befremden hervorrief. Die abendlichen Gespräche mit anderen Kursteilnehmern hätten allerdings immerhin ein schwaches Licht ins homöopathische Dunkel gebracht. Im Gegensatz zu den Heilerfolgen, die er in seiner Praxis allmählich verzeichnen konnte, überzeugte ihn der Kurs aber nicht.

\section{„Ein großes Aha-Erlebnis“}

Ein weiteres Beispiel dieser Kategorie soll verdeutlichen, wie Unzulänglichkeiten in der Schulmedizin Ärzte offen für alternative Ansätze machen können. Eine deutsche Kinderärztin schreibt in ihrem Werdegang, dass sie typische Kinderkrankheiten wie Bronchitis, Ohren- oder Mandelentzündungen in der DDR ausschließlich mit Antibiotika behandeln musste. Obwohl sie mit dieser einseitigen und langfristig erfolglosen Therapie unzufrieden war, durfte sie aufgrund der Gesetzeslage keine homöopathischen Arzneimittel einsetzen. ${ }^{4}$ Bereits im Medizinstudium sei ihr immer wieder gesagt worden, dass Homöopathie und andere alternative medizinischen Richtungen Hokuspokus seien. Besonders hervorgetan habe sich dahingehend der Gerichtsmediziner Otto Prokop (1921-2009), der „alle alternativen Heilmethoden auf das Schlimmste verteufelt" (SH 6) habe (vgl. [5, 6]).

Nach der Wende, als ihrer Neugier nichts mehr im Wege stand, besuchte sie einen Homöopathie-Kurs. Dieser Kurs sei für sie „ein großes Aha-Erlebnis“ gewesen, da die Dozenten sie restlos von dieser Heilmethode überzeugen konnten. Bestätigung habe sie dann wenig später im Arztalltag erfahren, als sie einen kleinen Jungen mit Angina und einer Penicillin-Allergie erfolgreich homöopathisch behandelte.

\section{Kategorie II: Unmittelbare Krankheitserfahrung}

Die zweitgrößte Gruppe des Samples stellen diejenigen Homöopathen dar, die die positiven Auswirkungen der Homöopathie nicht nur von außen beobachteten, sondern am eigenen Körper und dem ihrer Kinder erfahren haben. Entsprechende Angaben machten 10 Autoren (39\%).

\section{„Einfach nur begeistert"}

Eine Gynäkologin beispielsweise gibt an, nach der Geburt ihrer ersten Tochter schwer erkrankt zu sein, „ohne richtig Hilfe von der Schulmedizin erhalten zu haben“ (SH 1). Dieses einschneidende Erlebnis führte dazu, dass sie sich für Naturheilverfahren zu interessieren begann und den Entschluss fasste, sich mit einer eigenen Praxis niederzulassen.

„Langfristig wollte ich eine Praxis für Naturheilpraxis [sic] aufmachen und konnte mir vorstellen, dass Patienten auch nach homöopathischen Therapien fragen würden. Da dies ja alles Quatsch sei, entschied ich mich, einen Kurs für Homöopathie zu machen, um den Patienten Argumente zu geben, warum ich diese Therapie nicht mache. Ich suchte mir einen passenden Kurs [...]. Ich nahm an diesem Kurs teil mit der Zielsetzung, Argumente gegen die Homöopathie zu finden, und war sehr erstaunt, dass es eine Medizin geben soll, die das Thema ,Ähnliches mit Ähnlichem“ hatte“ (SH 1).

Die Aussage ist deshalb interessant, weil die Ärztin trotz ihrer Affinität für alternative Heilverfahren der Homöopathie gegenüber zunächst skeptisch bis ablehnend eingestellt war. Sie belegte den Kurs nicht in der Absicht, das dort erlernte Wissen zugunsten ihrer Patienten anzuwenden, sondern mit der Intention, deren Wünsche und Bedürfnisse als ungerechtfertigt zu widerlegen. Wider Erwarten brachte das Gehörte ihre Meinung aber ins Wanken. Statt ihre Vorannahmen zu bestätigen, wurden sie und andere Kursteilnehmer zum Nachdenken angeregt:

„Entweder ist das alles, was da vorgetragen wird, Quatsch und guruhaft oder kann es sein, dass es da eine wirksame Therapie gibt, über die an der Uni geschwiegen wird“ (SH 1)?

Um eine Antwort zu finden und da sich ihr gesundheitlicher Zustand zur selben Zeit erneut verschlechterte, begab sie sich in homöopathische Behandlung. Die Therapie schlug an, auch bei ihrem Mann und ihren Kindern, die der Homöopath ebenfalls erfolgreich behandelte. Daraufhin habe sie eine Ausbildung zur Homöopathin begonnen, da sie von der Homöopathie „einfach nur begeistert“ (SH 1) gewesen sei. Argumente gegen die Homöopathie habe sie bis heute keine gefunden. 


\section{„Sehr beeindruckend“}

Dass einige Autoren anfangs skeptisch waren, ob die Homöopathie tatsächlich helfen kann, geht auch aus anderen Berichten hervor. Ein Kinderarzt hatte Schwierigkeiten, die rezidiven fieberhaften Erkrankungen (Angina und Mittelohrentzündung) seines Sohns in den Griff zu bekommen. Seine Frau habe ihn dann gedrängt, sich nach medizinischen Alternativen umzuschauen, woraufhin er beim Zentralverband der Ärzte für Naturheilverfahren einen Homöopathie-Kurs besuchte. Obwohl ihm diese Lehre aufgrund seiner „schulmedizinischen Ausbildung und dem damit verbundenen linearen naturwissenschaftlichen Denken eher suspekt" (SH 4) vorkam, entdeckte er viele Gemeinsamkeiten zwischen einem Arzneimittelbild (Pulsatilla) und den Krankheitssymptomen seines Sohns. Kurze Zeit später bot ihm ein Rückfall Gelegenheit, dieses Mittel auszuprobieren. Zwar habe er geschwankt, sich dann aber doch zur einmaligen Gabe von Pulsatilla D12 entschlossen. Tatsächlich verschwand daraufhin das Fieber, einen Tag später sogar die eitrige Absonderung. Über diesen Heilerfolg schreibt der Arzt rückblickend:

„Das war zwar (für mich als,Naturwissenschaftler') ein Einzelfall, insgesamt aber doch sehr beeindruckend, so etwas wie ein Schlüsselerlebnis, so dass ich meine Ausbildung [...] fortsetzte. Von dieser Behandlung an gab es bei meinem Sohn keine einzige Otitis oder Angina mehr - das überzeugte noch mehr!“”

\section{„Offen für Neues“}

Die Erkrankung des Kindes machte die verzweifelten Eltern „offen für Neues“ (SH 25) - wie eine andere Ärztin ihre Reaktion auf die erfolglose konventionelle Behandlung ihres Kindes bezeichnet - und brachte sie indirekt mit der Homöopathie in Kontakt. Der Heilerfolg räumte nicht nur die anfänglichen Widerstände und Vorurteile aus dem Weg, sondern führte auch dazu, dass der betreffende Kinderarzt die Homöopathie-Ausbildung fortsetzte und rund 10 Jahre später eine eigene Praxis für Homöopathie eröffnete.

Als eigentlichen Impuls für die Hinwendung zur Homöopathie nennt eine Pastorin und Heilpraktikerin die „eigene Betroffenheit“ (SH 16). Die Schwangerschaft hätte sie veranlasst, sich stärker mit Medizin auseinanderzusetzen, da sie „nicht einfach nur die Patientin sein [wollte], die sich in die Hände von Spezialisten begibt" (SH 16). Um sich aus ihrer Unmündigkeit zu befreien, las sie populärwissenschaftliche Bücher zu medizinischen Fragen, ließ ihre Kinder zunächst aber schulmedizinisch behandeln. Beim ältesten Sohn, der in den ersten beiden Lebensjahren immer wieder an Bronchitis, Mittelohrentzündungen und Ekzemen erkrankte, halfen langfristig jedoch weder Antibiotika noch Cortison. Ein lebensgefährlicher Fieber- krampf habe sie schließlich zum Umdenken und zur Alternativmedizin bzw. Homöopathie gebracht. Diese Erfahrung teile sie mit vielen anderen Menschen, die sie später bei Fortbildungen kennen gelernt habe:

„Sie oder ein Familienangehöriger waren ernstlich erkrankt gewesen, hatten alle schulmedizinische Therapien hinter sich und fanden schließlich Heilung oder Besserung im alternativmedizinischen Bereich“(SH 16).

\section{Kategorie III: Schlüsselerlebnis, Initialmoment}

In ihren Berichten gaben 3 Homöopathen (11\%) ein bekehrendes „Aha-Erlebnis“( $\mathrm{SH}$ 6) an, das sie von der Wirksamkeit der Homöopathie überzeugte und den Ausschlag gab, sich fortan näher mit dieser Heilmethode zu beschäftigen. Ein solches Erlebnis war in den meisten Fällen eine positiv verlaufene Fallgeschichte. Sie wirkte auf die Autoren umso beeindruckender, als alle konventionellen Therapiemöglichkeiten bereits ausprobiert waren und eine Heilung deshalb hoffnungslos schien. Der Versuch, die Erkrankung homöopathisch zu behandeln, war der sprichwörtlich letzte Strohhalm, um den Kranken zu helfen.

\section{Ein Wunder für 7,50 DM}

So berichtet der ehemalige Tierarzt, dass er Anfang der 1990er Jahre während seiner Zeit als Assistent im Veterinäranatomischen Institut der Universität Leipzig mit der Behandlung eines Neufundländers betraut war, der an einer bakteriellen Entzündung beider Ohren litt. Bislang schlugen keine Antibiotika an, ebenso blieb eine operative Erweiterung beider Gehörgänge wirkungslos. Da der Hund starke Schmerzen hatte, wurde darüber nachgedacht, ihn einzuschläfern.

Der Tierarzt erinnerte sich an einen Kollegen, der in der Vergangenheit Erfolge mit homöopathischen Behandlungen hatte. Trotz Vorbehalten schilderte er ihm die Symptome der Entzündung, woraufhin der Kollege zum täglichen Verabreichen einiger Tropfen Hepar sulfuris C 30 riet. Das Befinden des Hundes besserte sich danach zusehends, bereits nach 2 Wochen war er „völlig wiederhergestellt“ (SH 27). Die Genesung, die in den Augen der Besitzerin an ein Wunder grenzte und noch dazu nur 7,50 DM Behandlungskosten verursachte, machte so großen Eindruck auf den Tierarzt, dass er alle seine verbliebenen Tiermedizin-Lehrbücher in ein Antiquariat trug, sich vom Erlös ein Künzli-Repertorium sowie eine homöopathische Arzneimittellehre besorgte und „beschloß, von nun an die richtige Medizin zu studieren“( $\mathrm{SH} 27)$. 


\section{„Verblüffende Parallelen“ zur modernen Physik}

Das ausschlaggebende Schlüsselerlebnis ist häufig an außergewöhnliche Heilerfolge wie dem gerade geschilderten geknüpft, kann aber auch auf bloßen Argumenten bezüglich der Vorzüge der Homöopathie beruhen. Ein diplomierter Physiker berichtet über seinen Weg zur Homöopathie, dass er nach dem Studium zunächst Zivildienst in einem Krankenhaus leistete. Dort lernte er die moderne Medizin kennen und erlebte den aus seiner Sicht fragwürdigen Umgang mit Kranken und Krankheiten. Beides schien ihn nachhaltig beeinflusst zu haben, denn er informierte sich über diverse alternativmedizinische Ansätze. An die Homöopathie dachte er dabei zunächst gar nicht. Einen entsprechenden Grundkurs belegte er lediglich zur Überbrückung der Zeit, die er benötigte, um klarere Vorstellungen zu bekommen. Umso überraschender und überzeugender wirkte das dort Gehörte auf ihn. Der Ansatz sei, so schreibt er, völlig kompatibel mit seinen Ansichten gewesen, „wie mit dem Thema Krankheit umgegangen werden müsse“( $\mathrm{SH} 8$ ). Darüber hinaus habe er „verblüffende Parallelen“ (SH 8) zur modernen Physik entdeckt, etwa die Kausalbeziehung, Wechselwirkungen oder die Einbindung des Beobachters. Nach und nach hätte die Physik an Reiz verloren, die Homöopathie ihn dagegen gefesselt. Das sei auch der Grund gewesen, sich tiefergehend mit der Heilmethode auseinanderzusetzen und eine Ausbildung zum Heilpraktiker zu absolvieren.

\section{Kategorie IV: Selbstverständnis als Arzt und Heilpraktiker}

Die 3 vorgestellten Kategorien dienen der besseren Einordnung der verschiedenen Beweggründe. Sie lassen bestimmte Grundmuster erkennen, denen die Hinwendung zur Homöopathie vorausgeht. Das bedeutet nicht zwangsläufig, dass die einzelnen Biographien teleologisch verlaufen: Das Bekenntnis zur Homöopathie stand nicht von Anfang an fest-auch wenn einige Umfrageteilnehmer das rückblickend gerne so darstellen. Eine Teilnehmerin beschreibt ihren Weg zur Homöopathie als

„eine stetige Suchbewegung in meinem Leben, die rückblickend stimmig in die Tätigkeit einer niedergelassenen Hausärztin mit Schwerpunkt klassische Homöopathie mündete“( $\mathrm{SH} 2)$.

Angeklungen ist bereits, dass manche Ärzte stattdessen der Homöopathie anfangs mit ausgesprochener Skepsis und Ablehnung begegneten.

\section{„Was ich schon immer gesucht hatte“}

Zwei Berichten (8\%) kann nun entnommen werden, dass nicht nur Unzufriedenheit und Heilerfolge für die Hinwendung zur Homöopathie verantwortlich sind. Ausschlaggebend für das Interesse an Homöopathie bzw. Alternativmedizin kann zudem das spezifische Selbst- und Krankheitsverständnis sein. Am deutlichsten zum Ausdruck kommt dies im Bericht einer ärztlichen Homöopathin. Den eigentlichen Impuls, sich von der Schulmedizin abzuwenden, gab die erfolglose Cortison-Behandlung ihres erkrankten Sohns. Statt ihm zu helfen, schwächte das Cortison zusehends das Immunsystem. Eine Freundin riet ihr zur Homöopathie - der weitere Verlauf folgt dem in Kategorie II thematisierten Muster. Die Ärztin beschreibt ihre Haltung gegenüber der Homöopathie nun folgendermaßen:

„Das war es, was ich schon immer gesucht hatte! Eine ganzheitliche Methode, die mir Mittel in und an die Hand gab, um ohne Nebenwirkungen zu therapieren! Trotz der anfänglichen Irritation durch das völlig andere Denkmodell der Homöopathie reizte mich vor allem die Herausforderung, das passende Mittel immer wieder neu zu finden, was sich von den starren Behandlungsschemata der Schulmedizin erfreulich abhob“ (SH 25). 
Die Homöopathie kommt also ihrem Wunsch entgegen, sich tiefergehend mit den Beschwerden der Patienten auseinanderzusetzen und individuell zugeschnittene Arzneimittel zu finden. Bislang war ihr das nicht möglich, die konventionellen Therapiemöglichkeiten beschränkten sich auf die pauschale Verordnung von Antibiotika oder Virostatika bei Infektionskrankheiten bzw. anderen synthetischen oder pflanzlichen Mitteln bei leichteren Erkrankungen.

Zusätzlich aufschlussreich für die mehrdimensionale Arzt-Patient-Beziehung sind ihre Ausführungen zur Doppelfunktion homöopathischer Medikamente:

„Für mich als Ärztin und Psychotherapeutin hat die Homöopathie noch einen besonderen Stellenwert. Das energetische Prinzip der Homöopathie, komprimiert in Form der Globuli, ist für mich Bindeglied zwischen der rein materiellen Sicht- und Behandlungsweise der ,Schulmedizin' und der Beschäftigung ausschließlich mit der seelisch-geistigen Dimension in der Psychotherapie. [...] Homöopathische Mittel, die ich verabreiche, sehe ich u.a. als wichtige Übergangsobjekte, die greifbar, fassbar sind, um dem Patienten zu vermitteln, dass er in seiner individuellen Lebenssituation und Erkrankung verstanden wird“ (SH 25).

\section{Das Gespräch}

Dieser Vorstellung zufolge materialisieren die Globuli die Aufmerksamkeit und Zuwendung des Arztes, die zuvor schon in ausführlichen Gesprächen verbal vermittelt worden ist. Man könnte dagegenhalten, dass das ja auch bei der Schulmedizin der Fall ist. Allerdings fehlen hier die zeitintensive Anamnese und das dadurch mögliche Vordringen zur eigentlichen Krankheitsursache als Vorbedingung der medikamentösen Behandlung.

Der wirksamste Teil der homöopathischen Therapie ist demnach das ausführliche Gespräch zwischen Arzt und Patient. Auch wenn andere Umfrageteilnehmer nicht so weit gehen, stimmen sie dieser Auslegung doch insoweit $\mathrm{zu}$, als sie die Bedeutung der homöopathischen Anamnese für den Heilprozess betonen. Eine Kinderärztin schreibt beispielsweise, bereits das erste Gespräch hätte eine Vertrauensbasis zwischen ihr und der Mutter bzw. dem Kind geschaffen, wie sie früher erst nach längerer Zeit möglich gewesen sei. Die Kombination von Schulmedizin und Homöopathie hätte sie deshalb als „enorme Bereicherung und ein Geschenk“ empfunden (SH 22). Eine Zahnärztin bekundet in ihrer Zuschrift, dass sie über die Homöopathie gelernt habe, „Menschen und besonders Kinder schneller und verständnisvoller zu erfassen“ (SH 23).

Die Homöopathie bietet also auch in Bezug auf Patientenkommunikation und empathische Gesprächsführung Lösungen an, die von der Schulmedizin lange Zeit als zweitrangig vernachlässigt worden sind. Je nach Gewichtung können sie ebenfalls ausschlaggebend dafür sein, dass sich ein Arzt von der Schulmedizin ab- und der Homöopathie zuwendet. Erinnert sei in diesem Zusammenhang nochmals an die Kategorie III zitierte österreichische Ärztin. An der Homöopathie schätzt sie besonders die Bedeutung des biographisch orientierten Arztgesprächs, das einem hilft, sich den Lebensphänomenen und dem realen Sein der Patienten zu öffnen (SH 13).

\section{Stabilisierung der Lebenskraft}

Einige Umfrageteilnehmer betonen letztlich, dass sie sich mit der Homöopathie gerade wegen des zugrundeliegenden Verständnisses von Gesundheit und Krankheit identifizieren können. Einem Kinderarzt gefällt beispielsweise, dass dem Organismus - eine rechtzeitige homöopathische Behandlung vorausgesetzt - durch die Vermeidung von konventionellen Medikamenten die Möglichkeit gegeben wird, ,sich aus sich heraus zu stabilisieren“ (SH 4). Ihm stimmt implizit eine weitere Ärztin zu. Sie schreibt in ihrem Bericht, dass es sie reizen würde, den Impuls zu finden, „der mit der Lebenskraft des Patienten in Resonanz tritt und damit die Selbstheilungskräfte des Organismus in Gang setzt“ (SH 25). Eine dritte Ärztin kritisiert ebenfalls die Schwächung von Lebenskraft und Vitalität durch die Einnahme von Antibiotika und Immunsuppressiva (SH 21). Krankheiten entstünden, wenn die individuelle Lebenskraft aus dem Gleichgewicht geraten ist. Sie zu korrigieren ist Aufgabe der homöopathischen Arzneimittel. Obwohl nur die letzten beiden Ärztinnen die für die Gesundheit eines Menschen so wichtige Lebenskraft explizit erwähnen, dürfte davon auszugehen sein, dass ihre Sichtweise von den übrigen Homöopathen geteilt wird.

\section{Fazit}

Die Auswertung der biographischen Kurzberichte förderte interessante Einblicke in die Entscheidungsfindung von homöopathischen Ärzten und Heilpraktikern zu Tage. Gefragt nach den individuellen Beweggründen geben viele Homöopathen an, sie seien ganz pragmatisch durch selbst- oder miterlebte Heilerfolge auf dieses alternativmedizinische Verfahren aufmerksam geworden. Die Linderung anhaltender und deshalb auch psychisch belastender Beschwerden kommt einer Offenbarung gleich, die Ärzte wie Laien zum Nachdenken und schließlich zur ernsthaften Auseinandersetzung mit der Homöopathie veranlasst(e). Auffallend ist, dass sich unter jenen „Konvertiten" auch solche befinden, die diesem Heilverfahren anfangs skeptisch bis ablehnend gegenüberstanden, sich von den Vorzügen aber überzeugen ließen. Das spricht wiederum für die besondere Bedeutung von Werbe-Vorträgen und Einführungskursen, mit denen sich nicht nur 
künftige Nutzer, sondern auch professionelle Anwender der Homöopathie ansprechen und gewinnen lassen.

Die meisten Homöopathen finden - so das zentrale Ergebnis der Untersuchung - jedoch durch die Unzufriedenheit mit der konventionellen Medizin bzw. dem offiziellen Gesundheitssystem zur Homöopathie. Im Schnitt vergehen 7 bis 8 Jahre, bis sich ein Arzt oder Laie nach seinem Studienabschluss dazu entscheidet, das nötige zusätzliche therapeutische Wissen zu erlernen. In diese Jahre fallen die negativen Erfahrungen mit dem begrenzten Methodenspektrum der Schulmedizin, das sich gerade bei chronischen Erkrankungen auf das Verordnen von symptomlindernden Medikamenten beschränkt. Auch in akuten Fällen bleibt Ärzten oft nichts anderes übrig, als Antibiotika zu verschreiben. Umso anziehender wirkt dann das Versprechen der Homöopathie, eine echte und noch dazu sanfte Alternative zu bieten. Hinzu kommen die Vorzüge dieser Heilmethode, die nicht primär auf die Wiederherstellung der Gesundheit gerichtet sind. Vor allem Kinder- und Hausärzte schätzen den ganzheitlichen und kommunikativen Ansatz der Homöopathie, der es ihnen ermöglicht, eine vertrauensvolle Atmosphäre zu schaffen und empathisch-fürsorglich auf den Patienten und dessen Beschwerden einzugehen. ${ }^{5}$

Insofern besitzt auch heute noch ein Aphorismus Gültigkeit, der den Nachruf auf einen 1962 verstorbenen homöopathischen Arzt schmückte [11] (S. 188):

„Wie so oft sehen wir, daß der echte homöopathische Arzt aus den Widersprüchen der klinischen Theorie und ärztlichen Praxis in die Homöopathie gerät und hier festen therapeutischen Boden gewinnt.“

\section{Online zu finden unter:}

http://dx.doi.org//10.1055/s-0043-111007

\section{.. Anmerkungen}

1 Da sich eine gendergerechte Schreibweise nicht immer einhalten lässt, werde ich im Folgenden die maskuline Form der Berufsund Statusbezeichnungen verwenden. Ich möchte aber ausdrücklich darauf hinweisen, dass damit sowohl Ärztinnen und Heilpraktikerinnen als auch weibliche Kranke, Patientinnen und Nutzerinnen alternativmedizinischer Methoden gemeint und eingeschlossen sind.

2 Maßgeblich beteiligt an besagtem Aufruf sowie der Korrespondenz mit den teilnehmenden Ärzten und Heilpraktikern war Sandra Dölker, Archivarin am IGM.

3 Anfang 2016 gehörten den insgesamt 40 Zweigvereinen der Hahnemannia 2225 (79\%) weibliche und lediglich 596 (21\%) männliche Mitglieder an.

4 Zur Geschichte der Homöopathie in der DDR siehe [4].

5 Robert Frank [1] untersucht in einer Studie, ob und inwieweit ärztliche Homöopathen auf das Diagnostik- und Therapiespektrum der konventionellen Medizin zurückgreifen.

\section{•• Literatur}

[1] Frank R. Integrating homeopathy and biomedicine: medical practice and knowledge production among German homeopathic physicians. Sociology of Health \& Illnes 2002; 24: 796-819. Online verfügbar unter: http://onlinelibrary.wiley.com/doi/10.1111/ 1467-9566.00319/full

[2] Identity Foundation. Gesundheitsorientierung und Gesundheitsvorsorge. Online verfügbar unter: http://www.ifd-allensbach.

Daniel Walther, M. A.

Karl-Metz-Str. 3

69115 Heidelberg

daniel.walther85@gmx.de

Jahrgang 1985, Studium der Geschichte/Germanistik mit Schwerpunkt Medizingeschichte, derzeit Promotion am Institut für Geschichte der Medizin der Robert Bosch Stiftung in Stuttgart über die homöopathische Laienbewegung in Deutschland seit 1870; Beschäftigung im Rahmen eines Postdoc-Projekts mit der Frage, warum deutsche und englische Ärzte in ihrer Praxis Homöopathie anwenden.

de/uploads/tx_studies/6184_Gesundheitsorientierung.pdf

[3] Kickbusch I. Die Gesundheitsgesellschaft: Megatrends der Gesundheit und deren Konsequenzen für Politik und Gesellschaft. Werbach-Gamburg: Verlag für Gesundheitsförderung; 2006

[4] Nierade A. Homöopathie in der DDR. Die Geschichte der Homöopathie in der Sowjetischen Besatzungszone und der DDR 1945 bis 1989. Essen: KVC-Verlag; 2012

[5] Prokop O. Medizinischer Okkultismus: Paramedizin. 2. Aufl. Stuttgart: G. Fischer; 1964

[6] Prokop O, Wimmer R. Der moderne Okkultismus: Parapsychologie und Paramedizin; Magie und Wissenschaft im 20. Jahrhundert. Stuttgart: G. Fischer; 1976

[7] Robert Koch-Institut, Hrsg. Daten und Fakten: Ergebnisse der Studie "Gesundheit in Deutschland aktuell 2012“. Beiträge zur Gesundheitsberichterstattung des Bundes. Berlin: RKI; 2014: Online verfügbar unter: http:// www.geda-studie.de/deutsch/ergebnisse/geda-2012.html
[8] de Sombre S. Bekanntheit, Verwendung und Image homöopathischer Arzneimittel. Ergebnisse einer bevölkerungsrepräsentativen Befragung. Homöopathie Forum; Organisation klassisch homöopathisch arbeitender Heilpraktiker e.V.; 2009: Online verfügbar unter: http://www.homoeopathie-forum.de/ anhaenge/sonstige_anhaenge/BAH_Praesentation_Homoeopathie_handout.pdf

[9] de Sombre S. Homöopathische Arzneimittel 2014. Bekanntheit, Verwendung und Image. Ergebnisse einer bevölkerungsrepräsentativen Befragung. Bundesverband der Arzneimittel-Hersteller e. V; 2014: Online verfügbar unter: https://www.bah-bonn.de/ index.php?elD=dumpFile\&t $=$ $\mathrm{f} \& \mathrm{f}=4233$ \&token $=8724 \mathrm{~d} 36 \mathrm{ab} 66153$ 21300b76f723126d5e07d6e21e

[10] Statistik der Bundesärztekammer 2016. Online verfügbar unter: http://www.bundesaerztekammer.de/fileadmin/user_upload/downloads/pdf-Ordner/Statistik2016/ Stat16AbbTab.pdf

[11] Unger H. Dr. phil. Georg Hermann Robert Kerl †. AHZ 1962; 207: 187-188 